\title{
Silencing Smad7 potentiates BMP2-induced chondrogenic differentiation and inhibits endochondral ossification in human synovial-derived mesenchymal stromal cells
}

Pengcheng Xiao, Zhenglin Zhu, Chengcheng Du, Yongsheng Zeng, Junyi Liao, Qiang Cheng, Hong Chen, Chen Zhao* and Wei Huang ${ }^{*}$ (D)

\begin{abstract}
Background: Bone morphogenetic protein 2 (BMP2) is a promising chondrogenic growth factor for cartilage tissueengineering, but it also induces robust endochondral ossification. Human synovial-derived mesenchymal stromal cells (hSMSCs) have attracted great interest due to their poor potential for differentiation into osteogenic lineages. Smad7 plays a significant in the endochondral ossification. In this study, we explored a new method to amplify the BMP2-induced chondrogenic differentiation of hSMSCs by downregulating Smad7 and applying a cellular scaffold.

Methods: hSMSCs were isolated from human knee joint synovium from 3 donors through adhesion growth. In vitro and in vivo models of the chondrogenic differentiation of hSMSCs were established. Transgenic expression of BMP2 and silencing of Smad7 and Smad7 was achieved by adenoviral vectors. The osteogenic differentiation was detected by alkaline phosphatase staining, alizarin red staining, and RT-PCR analysis of the osteogenic genes RUNX2, Osterix, and Osteocalcin. The chondrogenic differentiation was detected by Alcian blue staining and RT-PCR analysis of the chondrogenic genes SOX9, COL2, and aggrecan. Hypertrophic differentiation was detected by the markers COL10 and MMP13. A subcutaneous stem cell implantation model was established with polyethylene glycol citrate-co-N-isopropylacrylamide (PPCN) scaffolds and athymic nude mice (3/group, 4-6 week-old female) and evaluated by micro-CT, H\&E staining, and Alcian blue staining. An immunohistochemistry assay was used to detected COL1 and COL2, and an immunofluorescence assay was used to detect COL10 and MMP13.

Results: These hSMSCs identified by flow cytometry. These hSMSCs exhibited lower osteo-differentiation potential than iMads and C3H10T1/2-cells. When Smad7 was silenced in BMP2-induced hSMSCs, the chondrogenic differentiation genes SOX9, COL2, and aggrecan were enhanced in vitro. Additionally, it silencing Smad7 led to a decrease in the hypertrophic differentiation genes COL10 and MMP13. In subcutaneous stem cell implantation assays, immunofluorescence and immunohistochemical staining demonstrated that silencing Smad7 increased the number of COL2-positive cells and decreased the expression of COL1, COL10, and MMP13.

(Continued on next page)
\end{abstract}

\footnotetext{
*Correspondence: Shawn95@yeah.net; huangwei68@263.net Department of Orthopedics, The First Affiliated Hospital of Chongqing Medical University, Chongqing 400016, China
}

C C The Author(s). 2021 Open Access This article is licensed under a Creative Commons Attribution 4.0 International License, which permits use, sharing, adaptation, distribution and reproduction in any medium or format, as long as you give appropriate credit to the original author(s) and the source, provide a link to the Creative Commons licence, and indicate if changes were made. The images or other third party material in this article are included in the article's Creative Commons licence, unless indicated otherwise in a credit line to the material. If material is not included in the article's Creative Commons licence and your intended use is not permitted by statutory regulation or exceeds the permitted use, you will need to obtain permission directly from the copyright holder. To view a copy of this licence, visit http://creativecommons.org/licenses/by/4.0/. The Creative Commons Public Domain Dedication waiver (http://creativecommons.org/publicdomain/zero/1.0/) applies to the data made available in this article, unless otherwise stated in a credit line to the data. 
(Continued from previous page)

Conclusion: This study suggests that the application of hSMSCs, cell scaffolds, and silencing Smad7 can potentiate BMP2-induced chondrogenic differentiation and inhibit endochondral ossification. Thus, inhibiting the expression of Smad7 in BMP2-induced hSMSC differentiation may be a new strategy for cartilage tissue-engineering.

Keywords: Human synovial-derived mesenchymal stromal cells, BMP2, Smad7, PPCNg, Chondrogenic differentiation, Endochondral ossification

\section{Introduction}

The autologous repair capacity of the articular cartilage is very limited, because of the low mitotic activity of articular chondrocytes and the absence of the blood vessels to deliver putative precursor cells [1]. Tissueengineering-based treatment strategies that include growth factors, seed cells, and implant scaffolds may open new avenues for the treatment of cartilage injuries $[2,3]$. Many different growth factors, such as transforming growth factor beta (TGF- $\beta$ ), bone morphogenetic proteins (BMPs), and fibroblast growth factors (FGFs), have been shown to have the ability to promote stem cell chondrogenic differentiation [4, 5]. Among these factors, bone morphogenetic protein 2 (BMP2), a member of the TGF- $\beta$ superfamily, has shown great potential for inducing the chondrogenic differentiation, osteogenic differentiation, and endochondral ossification of MSCs $[6,7]$. As BMP2 has been approved by the United States Food and Drug Administration (FDA) and is widely used in spine surgery, its biosafety has been proven $[8,9]$. Our previous study indicated that BMP2 is capable of promoting MSC chondrogenic differentiation, chondrocyte proliferation, and hypertrophic differentiation [10, 11]. A recent study by Chan et al. found that localized codelivery of BMP2 and soluble VEGFR1 (sVEGFR1) skewed the differentiation of resident skeletal stem cells towards the articular cartilage [12]. All of these studies suggested that BMP2-induced cartilage formation can be a promising solution for cartilage defect repair.

However, the use of BMP2 for bioengineered cartilage construction is still under debate, because BMP2 promotes not only chondrogenic differentiation but also endochondral ossification. Previous studies showed that hypertrophic markers, such as COL10 and MMP13, were expressed in the BMP2-induced ectopic cartilagelike mass at 21 days [13]. Vascular invasion, matrix calcification, and endochondral ossification were also observed in BMP2-induced MSCs, leading to the destruction of chondrogenic phenotypes [11, 14]. Therefore, endochondral ossification is the main obstacle to BMP2-induced cartilage formation $[10,11]$. Thus, it is conceivable that BMP2 may be used for clinical cartilage repair if BMP2-induced chondrogenesis hypertrophy and endochondral ossification could be inhibited. To obtain a stable chondrogenic phenotype, many efforts have been made to inhibit the hypertrophic differentiation and endochondral ossification induced by BMP2.

Recently, human knee joint synovial-derived mesenchymal stromal cells (hSMSCs) have been reported to be one of the most ideal types of MSCs for cartilage regeneration research because they possess certain advantages, including the tissue specificity, abundant sources, powerful regenerative capabilities, and stable pluripotent differentiation potential $[15,16]$. Mesenchymal stromal cells can easily be isolated from different tissues and stimulated by cytokines, leading to an upregulation of molecules typical of articular cartilage [17, 18]. In addition, it has been reported that synovium-derived stromal cells have relatively poor potential to differentiate into the osteogenic lineage in vitro $[19,20]$. BMP2 has been demonstrated to govern the divergence of chondrogenesis in hSMSCs [17]. However, hSMSCs are also reported to be capable of endochondral ossification differentiation in vivo and thus can also impair chondrogenesis [18].

To further inhibit BMP2-induced endochondral ossification, we aimed to adjust the expression of Smad7 during cartilage formation. In our previous study, we found that overexpression of BMP2 leads to the upregulation of Smad7 expression [9]. Smad7, which belongs to the inhibitory Smad family, antagonizes the TGF- $\beta$ /BMP signaling pathway through multiple mechanisms in both the cytoplasm and nucleus [21, 22]. Previous studies have shown that Smad7 is an inhibitor of BMP2-induced chondrogenic differentiation [23]. Moreover, Smad7 is essential for endochondral ossification [24]. In this study, we hypothesize that specific and stable BMP2-based tissue-engineered cartilage can be obtained by using hSMSCs with Smad7 expression knocked down. In addition, polyethylene glycol citrate-co- $N$-isopropylacrylamide (PPCNg), a biocompatible scaffold, was used to sustain cell attachment and proliferation [25]. We used hSMSCs infected with adenoviruses to investigate the effect of osteogenic and chondrogenic differentiation both in vitro and in vivo. We demonstrated that silencing Smad7 expression not only potentiated the chondrogenic differentiation induced by BMP2, but also inhibited the BMP2-induced hSMSC chondrogenesis hypertrophy. Therefore, our investigation provides another possible strategy for BMP2-based cartilage repair. 
These results will offer abundant practical evidence for BMP2-mediated bioengineered cartilage construction.

\section{Materials and methods Isolation and cell culture of hSMSCs}

This research was approved by the Research Ethics Committee of the First Affiliated Hospital of Chongqing Medical University. All the participants were patients from the Orthopedic Department of the First Affiliated Hospital of Chongqing Medical University, aged from 25 to 40 years old who underwent lower limb amputations. We obtained informed consent from all three patients prior to their participation.

Primary hSMSCs were grown from human synovial membrane samples from the knee joint as previously reported [26]. Briefly, the human synovial tissue was washed with phosphate-buffered saline (PBS) containing $1 \%$ penicillin and streptomycin 3 times in a sterile petri dish and then minced with sterile scissors into 1-2 mm fragments. Two or three synovial fragments were then plated in $25 \mathrm{~cm}^{2}$ flasks in $5 \mathrm{~mL}$ of Dulbecco's modified Eagle's medium (DMEM, Gibco, China) containing 20\% fetal bovine serum (FBS, Gibco, Australia) and 1\% antibiotic-antimycotic solution (Gibco, China) and cultured for 14 days. When the cells grew to approximately $80 \%$ confluency, the synovial fragments were removed, and the cells were passaged into T25 flasks and considered as passage 0 (P0). In each experiment, the medium was refreshed every 3 days. When the cells reached $90 \%$ confluency, they were divided at a 1:2 ratio. In subsequent experiments, only cells between passages 1 and 5 were used.

\section{Cells culture}

Immortalized mouse adipose-derived MSCs (iMADs) and $\mathrm{C} 3 \mathrm{H} 10 \mathrm{~T} 1 / 2$ cells were obtained from ATCC (Manassas, VA). As previously noted [27], MSCs were cultured in DMEM with 10\% fetal bovine serum (FBS), $1 \%$ penicillin, and streptomycin. The cells were grown in a monolayer under standard culture conditions $\left(37^{\circ} \mathrm{C}, 5 \%\right.$ $\left.\mathrm{CO}_{2}\right)$.

\section{Crystal violet assay and CCK-8 assays}

As reported [28], we seeded $10^{4} \mathrm{hSMSC}$ from passage 1 into each well of a 96-well culture plate and then cultured them for 11 days. At the indicated time-points, the cells were carefully washed with PBS and stained with $0.5 \%$ crystal violet/formalin solution at room temperature for $20-30 \mathrm{~min}$. The stained cells were washed with tap water and air-dried before capturing macrographic images. Each time-point included six replicate wells. For quantitative measurement, the stained cells were dissolved in 10\% acetic acid, and the optical density was measured at $590 \mathrm{~nm}$ as described [29].
To determine the change in cell proliferation ability with passaging, we used Cell Counting Kit-8 (CCK-8, MedChemExpress) to test hSMSCs at passages 1 and 5 $[30,31]$. The results were recorded by a microplate reader (Thermo Scientific ${ }^{\mathrm{Tm}}$, USA) at an absorbance of $450 \mathrm{~nm}$. The growth curves were drawn, and the cell proliferation activity was analyzed.

\section{Phenotypic identification of hSMSCs}

The identification of MSCs requires analyzing the expression of the relevant surface antigens [18]. hSMSCs were harvested and resuspended in PBS containing $1 \%$ bovine serum albumin at approximately $1 \times 10^{6}$ cells $/ \mathrm{mL}$. Then, $0.1 \mathrm{~mL}$ of the cell suspensions was incubated with conjugated CD73 (BioLegend), CD105 (BioLegend), CD90 (BioLegend), CD45 (BD Biosciences), CD44 (BioLegend), HLA-DR (BD Biosciences), CD14 (BD Biosciences), or CD34 (BD Biosciences) antibodies for $30 \mathrm{~min}$ at $4{ }^{\circ} \mathrm{C}$ in the dark. After being washed three times with PBS, the labeled cells were resuspended in $0.2 \mathrm{~mL}$ of PBS and analyzed with the CytoFLEX system (Beckman Coulter). The acquired data were analyzed by using CytExpert software (Beckman Coulter) [26, 28].

Briefly, to identify the multiple differentiation potential [32, 33], cells were seeded in a 6-well plate, and the differentiation medium was replaced when the cell density reached $60 \%$. Osteogenic medium was composed of DMEM with $10 \%$ FBS, $10 \mathrm{mM}$ bglycerophosphate (Sigma-Aldrich), $50 \mathrm{mg} / \mathrm{mL}$ ascorbate (Sigma-Aldrich), and $100 \mathrm{nM}$ dexamethasone (Sigma-Aldrich). The osteogenic differentiation results were observed at 7 days with the BCIP/NBT Alkaline Phosphatase Color Development Kit (Beyotime) and at 21 days with Alizarin Red S staining $(0.2 \%, \mathrm{pH}=$ 8.3) (Solarbio). In terms of chondrogenic differentiation, we used Synovial Mesenchymal Stromal Cell Chondrogenic Differentiation Basal Medium (Cyagen) to induce hSMSCs for 14 days and assessed them by Alcian Blue staining (1\%) (Solarbio) and immunohistochemical attaining of COL2 (Abcam, UK; 1:200). Adipogenic differentiation medium solution A consisted of DMEM complemented with 10\% FBS, 10 $\mathrm{mM}$ dexamethasone, $50 \mathrm{~g} / \mathrm{mL}$ indomethacin (Sigma), $45 \mathrm{mM}$ 3-isobutyl-1-methylxanthine (Sigma), and $10 \mathrm{~g} /$ $\mathrm{mL}$ insulin (Sigma). Adipogenic differentiation medium solution B consisted of DMEM supplemented with $10 \%$ FBS and $10 \mathrm{~g} / \mathrm{mL}$ insulin (Sigma). After incubation in solution A for 3 days, the medium was changed to solution B for another day. After the appearance of the morphologic features of differentiation, Oil Red $\mathrm{O}$ staining $(0.5 \%$ in isopropanol $)$ (Solarbio) was conducted to determine intracellular lipid droplet formation. 


\section{Recombinant adenovirus construction}

Recombinant adenoviruses were generated using AdEasy technology as described previously $[10,11]$. The coding regions of BMP2, Smad7, red fluorescent protein (RFP), and green fluorescent protein (GFP) were amplified with PCR and cloned into adenovirus shuttle vectors. Then, the vectors were utilized to generate recombinant adenoviruses in HEK 293 cells [34]. The siRNA target sites against the human Smad7-coding region were cloned into the pSES adenovirus shuttle vector to create recombinant adenoviruses [35]. The resulting adenoviruses were designated Ad-GFP, Ad-BMP2, Ad-Smad7, and Ad-siSmad7. Ad-GFP and Ad-RFP were used as vector controls.

\section{RNA isolation and quantitative PCR (qPCR)}

Total RNA was purified from cells in $60-\mathrm{mm}$ dishes using TRIzol (Invitrogen) according to the manufacturer's instructions [35]. Then, cDNA was obtained from total RNA extracted from cells using a reverse transcription (RT) reaction kit (TAKARA, Japan) [13, 36]. cDNA was completed with SYBR Premix Ex Taq ${ }^{\mathrm{mm} I I}$ (TaKaRa, Japan), and the program was carried out as follows: $95^{\circ} \mathrm{C}$ for $30 \mathrm{~s}$ for one cycle; then, $95^{\circ} \mathrm{C}$ for $5 \mathrm{~s}$ and $60^{\circ} \mathrm{C}$ for $30 \mathrm{~s}$, followed by plate reading for 40 cycles. The PCR primers (Table S1) were designed using Primer3 plus in Supplementary Table 1. The relative expression levels of the mRNAs in the groups were analyzed using the $2 \Delta \Delta C T$ method $[32,37]$.

\section{Western blot analysis}

Cells were seeded in six-well plates and treated per the experimental design. Total protein was obtained after lysis, and the cleared lysates were denatured by boiling for $10 \mathrm{~min}$ with $10 \%$ sodium dodecyl sulfatepolyacrylamide gel electrophoresis buffer. The proteins were separated by electrophoresis with Tris-glycine gels and carefully transferred onto polyvinylidene difluoride (PVDF) membranes in the dark. Then, the PVDF membranes were blocked with $5 \%$ evaporated milk for $1 \mathrm{~h}$ and incubated overnight with primary antibodies against BMP2 (Abcam, USA) and Smad7 (Santa Cruz, USA). After washing, the membranes were probed with a fluorescently labeled secondary antibodiesy. The immunereactive signals were detected using a Bio-Rad machine. In addition, the membranes were incubated with a monoclonal mouse anti-human $\beta$-actin (Abcam, USA) antibody as a loading control. The relative band intensity was measured using ImageJ analysis software $[35,36]$.

\section{Enzyme-linked immunosorbent assay}

The concentration of BMP2 in the supernatants of infected hSMSCs was examined with enzyme-linked immunosorbent assay (ELISA) kits (Neobioscience, China).
After $24 \mathrm{~h}$ of adenovirus infection, the supernatants of the two groups were collected and assayed according to the manufacturer's instructions. The assays were performed in the same way after infection for $48 \mathrm{~h}$ and $72 \mathrm{~h} \mathrm{[32].}$

\section{ALP staining and activity}

For ALP staining, cells were fixed with $4 \%$ paraformaldehyde for $30 \mathrm{~min}$. The cells were then washed twice with PBS and stained using the BCIP/NBT Alkaline Phosphatase Color Development Kit (Beyotime). Staining was observed under a bright field microscope after $30 \mathrm{~min}$ [32, 38].

For the measurement of ALP activity, cells were washed twice with PBS and lysed with $150 \mu \mathrm{L}$ of NP-40 lysis buffer (Beyotime). The cell lysates were quantified by an alkaline phosphatase assay kit (Beyotime) using pnitrophenyl phosphate (pNPP) as the substrate. In the presence of magnesium ions, pNPP was hydrolyzed by phosphatases to phosphate and p-nitrophenol. The rate of p-nitrophenol liberation is proportional to ALP activity and can be measured photometrically. The ALP activity was measured by a microplate reader (Thermo Scientific $^{\mathrm{Tw}}$, USA) at an absorbance of $405 \mathrm{~nm}$ [39]. In addition, the protein concentration of the cell lysate was determined by a BCA Protein Assay Kit (Beyotime), and ALP activity was normalized to total protein per well.

\section{Matrix mineralization assay (Alizarin Red S staining)}

Subconfluent hSMSCs, iMads, and C3H10T1/2 cells were infected with the indicated adenoviral vectors for 2 days. After adenovirus infection, the cells were cultured in the presence of ascorbic acid $(50 \mathrm{mg} / \mathrm{ml})$ and bglycerophosphate $(10 \mathrm{mM})$ for 14 days. The mineralization nodules were assessed by Alizarin Red $\mathrm{S}$ staining $[28,32]$. Briefly, the cells were fixed with paraformaldehyde at room temperature for $10 \mathrm{~min}$ and washed with PBS (pH adjusted to 4.2). The fixed cells were incubated in a $37^{\circ} \mathrm{C}$ incubator with $2 \%$ Alizarin Red $\mathrm{S}$ for $10 \mathrm{~min}$, followed by careful washing with distilled water. The calcium deposits were observed under a microscope. For quantification, Alizarin Red S was dissolved in $10 \%$ acetic acid and the absorbance was detected at $405 \mathrm{~nm}$ with a microplate reader [32]. Total DNA was purified from cells per well using TRIzol (Invitrogen) and measured by spectrophotometer (Thermo, NanoDrop) [35]. The results were normalized to the total DNA per well and at least three independent experiments were performed.

\section{The chondrogenic and hypertrophic differentiation protocol}

For the chondrogenic differentiation and hypertrophic differentiation of hSMSCs, the micromass culture method was used as previously noted $[9,10,13]$. Then, the cells were cultured in chondrogenic medium for 1 week, and 
this medium was composed of DMEM with ITS (SigmaAldrich), $50 \mu \mathrm{g} / \mathrm{mL}$ ascorbate (Sigma-Aldrich), $100 \mathrm{nM}$ dexamethasone (Sigma-Aldrich), and $10 \mathrm{ng} / \mathrm{mL}$ transforming growth factor- $\beta 3$ (Sigma-Aldrich). Then, the cells were cultured in hypertrophic medium for another week, and this medium contained ITS supplement, $50 \mu \mathrm{g} / \mathrm{mL}$ ascorbate, $1 \mathrm{nmol} / \mathrm{L}$ dexamethasone, and $100 \mathrm{ng} / \mathrm{mL}$ triiodothyronine (T3, Sigma-Aldrich) [39].

Briefly, hSMSCs were washed with PBS, treated with 4\% paraformaldehyde for $30 \mathrm{~min}$, and washed again with PBS, followed by staining with $0.5 \%$ Alcian blue in $0.1 \mathrm{M}$ $\mathrm{HCl}(\mathrm{pH} 1.0)$ for $12 \mathrm{~h}$. Then, cells were photographed with a microscope $[10,11]$.

\section{Immunohistochemical analysis of cells}

As previously reported [11], cell samples were fixed in 44\% paraformaldehyde for $10 \mathrm{~min}$ and washed with distilled water. Next, the cells were treated with $3 \% \mathrm{H}_{2} \mathrm{O}_{2}$ for $15 \mathrm{~min}$ at room temperature to eliminate the endogenous peroxidase activity and blocked with normal goat serum for $40 \mathrm{~min}$ at room temperature. Then, the cells were incubated with anti-Col2 (Abcam, UK; 1:200), anti-ColX (Santa Cruz Biotechnology; 1:200), and antiMMP13 (Abcam, UK; 1:200) primary antibodies overnight at $4{ }^{\circ} \mathrm{C}$. The sections were subsequently incubated with a secondary antibody, IgG-HRP (CST; 1:200). The resulting sections were photographed under a microscope.

\section{Stem cell implantation to ectopic cartilage formation and using of polyethylene glycol citrate-co- $N$ - isopropylacrylamide (PPCN) scaffolds in vivo}

Polyethylene glycol citrate-co- $N$-isopropylacrylamide (PPCN) was synthesized, as previously described [40]. PPCN powder was dissolved in PBS (at 100 $\mathrm{mg} / \mathrm{mL}$ ), sterilized by syringe filtration with $0.22 \mu \mathrm{m}$ filters, and stored at $4{ }^{\circ} \mathrm{C}$. Subcutaneous ectopic bone formation was carried out as previously reported [9-11].

Briefly, subconfluent hSMSCs were infected with specific adenoviruses until fluorescence could be seen and then harvested for subcutaneous injection. After washing with PBS, hSMSCs were resuspended in $5 \times$ $10^{6}$ cells in $100 \mu \mathrm{L}$ of PPCN scaffolds on ice. The cell/scaffold mixture was subcutaneously injected into the flanks of athymic nude mice (3 mice per group and 3 injections per mouse, 4-6 week-old female, the Experimental Animal Center, Chongqing Medical University, Chongqing, China). PPCNg alone and PPCNg with cells transduced with Ad-GFP, Ad-Smad7, or Ad-siSmad7 alone were used as controls. At 4 weeks, the animals were euthanized to harvest ectopic masses.
Micro-computed tomography $(\mu \mathrm{CT})$ analysis: hematoxylin \& eosin (H\&E) staining and Alcian blue staining

The retrieved masses were fixed with $10 \%$ PBSbuffered formalin and imaged using SkyScan1174 Xray microtomograph (micro-CT) (Bruker Company, Belgian). NRecon software was used for 3D image reconstruction, and all the image data analyses were performed using CT-AN software. The total volume (TV) and bone volume/total volume (BV/TV) were measured as described $[9,32]$.

After $\mu \mathrm{CT}$ imaging, the retrieved masses were decalcified, paraffin-embedded, and sectioned. The sections were stained with $\mathrm{H} \& \mathrm{E}$ and Alcian Blue as previously described $[9,11]$.

\section{Immunohistochemical and immunofluorescence stain assays}

In the immunohistochemical staining assay [41, 42], sections were deparaffinized with xylene, rehydrated using graded ethanol, treated with $3 \% \mathrm{H}_{2} \mathrm{O}_{2}$ for 10 min to inhibit the endogenous peroxidase activity, boiled in citrate buffer $(\mathrm{pH} 6.0)$ for $20 \mathrm{~min}$ at $95-100^{\circ} \mathrm{C}$, and blocked with normal goat serum. Then, the sections were incubated with primary antibodies against COL2 (Abcam, 1:200) and COL1 (Collagen 1a1, Abcam, 1:200) at $4{ }^{\circ} \mathrm{C}$ overnight. After being washed, the sections were incubated with biotin-labeled secondary antibodies for $30 \mathrm{~min}$, followed by incubation with streptavidin-HRP conjugate for $20 \mathrm{~min}$ at room temperature. Staining without primary antibodies was utilized as a negative control. All the images were obtained by using a microscope (Olympus).

In the immunofluorescence staining assay, the paraffin-embedded tissue sections were deparaffinized, rehydrated, and subjected to staining with an MMP13 antibody (Abcam, UK; 1:200) and Col10 antibody (Abcam, UK; 1:200) [32]. This was followed by incubation with corresponding fluorophore-conjugated antibodies. The immunohistochemical staining results were observed by inverted fluorescence microscopy (Olympus), and the images were analyzed using an Olympus auxiliary system.

\section{Statistical analysis}

All the data are representative of at least three experiments performed in triplicate, which yielded similar results, unless otherwise indicated. All the quantitative experiments were performed in triplicate and/or repeated through three independent batches of experiments. The statistical analyses were performed using the software package SPSS 14.0 and by one-way analysis of variance and Student's $t$ test to determine the significance of differences between results, with ${ }^{*} p<0.05$ and $* * 0<0.01$ being regarded as significant $[9,10]$. 


\section{Results}

Isolated hSMSCs that exhibit high proliferative activity hSMSCs are considered to have the greatest potential for cartilage regeneration research due to their tissuespecific advantages. Although hSMSCs have been used rather extensively, their biological features are very different due to age, arthritis, or other donor joint conditions $[18,26]$. Here, we isolated primary human synovial mesenchymal stromal cells from the knee joint synovial membrane of three donors.

After $72 \mathrm{~h}$, fibroblast-like primary cells migrated outwards from the minced fragments of synovial tissues (Fig. 1a). Primary cells grew relatively slowly, and after 14 days of culture in dishes, the cells covered the field of vision (Fig. 1a). The morphology of cells cultured to passages 1 and 5 (P1 and P5, respectively) showed a spindle-shaped appearance and plastic-adherent properties, and there was no distinct change at passage 5 (Fig. 1a). Crystal violet staining assays indicated that hSMSCs reached $70 \%$ confluence at day 5, while at day 9, they covered the culture plate (Fig. 1b). Quantitative assessment of the stained cells showed that cell proliferation plateaued on day 5 and continued to increase after day 7 (Fig. 1c).

We used Cell Counting Kit (CCK)-8 to detect the effect of passage on hSMSC proliferative activity. The results showed similar growth capacities of P1 and P5 cells and confirmed the crystal violet staining results showing that cell proliferation had a short plateau period after day 5 (Fig. 1d). hSMSCs express most MSC markers and are capable of multidirectional differentiation potential.

According to the criteria for mesenchymal stromal cell identification, we identified the surface markers of hSMSCs using flow cytometry [29, 32, 33]. Flow cytometric results showed that $\mathrm{P} 1 \mathrm{hSMSCs}$ were positive for the MSC markers CD73, CD90, CD44, and CD105 and weakly expressed the hematopoietic markers CD34, CD14, CD45, and HLA-DR, which indicated that P1 hSMSCs express most of the consensus MSC markers and suggested that these cells may possess MSC-like characteristics (Fig. 1e). The average mean fluorescence for all markers between the three donor populations is shown (Fig. 1f).

In addition, when cultured in osteogenic, adipogenic, or chondrogenic medium, hSMSCs could readily be induced to differentiate into osteogenic, adipogenic, and chondrogenic lineages, respectively. Osteogenic potential was confirmed by staining with alkaline phosphatase (ALP) (Fig. 1g, g1) and Alizarin Red (Fig. 1g, g2). Chondrogenic potential was confirmed by staining with sulfated glycosaminoglycans using Alcian blue and COL2 protein using immunohistochemical staining (Fig. 1g, g3, g4), while adipogenic potential was evaluated by observation of small cytoplasmic lipid droplets stained using
Oil Red O (Fig. 1g, g5). Osteogenic, chondrogenic, and adipogenic differentiation potential were also confirmed by RT-qPCR analysis of genes included the osteogenic relative factors RUNX2 and Osterix, the chondrogenic relative factors Sox9 and COL2, and the adipogenic relative factor PPAR- $\gamma$ (Fig. 1h).

\section{Recombinant adenovirus effectively overexpressed transgenes in hSMSCs for a relatively long-time}

We constructed recombinant adenovirus to stabilize overexpression of BMP2 and Smad7 using AdEasy technology, and we constructed another recombinant adenovirus that expressed small interfering RNA (siRNA) targeting the coding region of human Smad7 using the recently described established pSOS system [9, 10, 13]. To determine whether recombinant adenovirus can be effectively transduced into hSMSCs, we observed the expression of GFP and RFP by fluorescence microscopy $24 \mathrm{~h}$ after infection (Fig. 2a). RT-PCR confirmed that the transgenes were highly expressed in the hSMSCs that were infected with the respective adenoviral vectors for 3 and 5 days (Fig. 2b). Moreover, we found that BMP2 effectively upregulated Smad7 expression at 3 and 5 days after infection (Fig. 2c). Western blot results showed that the Ad-BMP2 group effectively upregulated the BMP2 protein levels at 3 days (Fig. 2d,e). BMP2 is a secretory protein, and we performed ELISA to further detect the protein concentration in the cell supernatant [6]. The results showed that the BMP2 concentration in the supernatant increased gradually with increasing time (Fig. 2f).

To determine whether these recombinant adenoviruses could effectively regulate Smad7 expression levels in hSMSCs, fluorescence microscopy and RT-qPCR were used. The effectively transduction of adenoviral vectors was observed $48 \mathrm{~h}$ after infection (Fig. $2 \mathrm{~g}$ ). We found that Ad-Smad7 greatly upregulated the mRNA expression of Smad7 compared with the control (Fig. 2h). In addition, Ad-siSmad7 effectively knocked down Smad7 mRNA expression in hSMSCs from 5 to 14 days (Fig. $2 \mathrm{i})$. At the protein level, western blot analysis revealed that the BMP2 and siSmad7 groups had lower expression levels of Smad7 than the BMP2 group and the BMP2 and Smad7 groups had higher expression levels than the BMP2 group (Fig. 2j, k).

MSC in vitro cultivation for clinical treatments may greatly affect MSC properties. A primary challenge is replicative senescence, which impairs MSC functions [43]. To protect the therapeutic potential of hSMSCs, we evaluated senescence after adenovirus transduction. After $72 \mathrm{~h}$ of adenovirus transfection, the cells were subjected to apoptosis assays (Fig. 2m) and beta galactosidase staining (Supplement, Figure 1), and no significant changes were observed compared with the control group. 


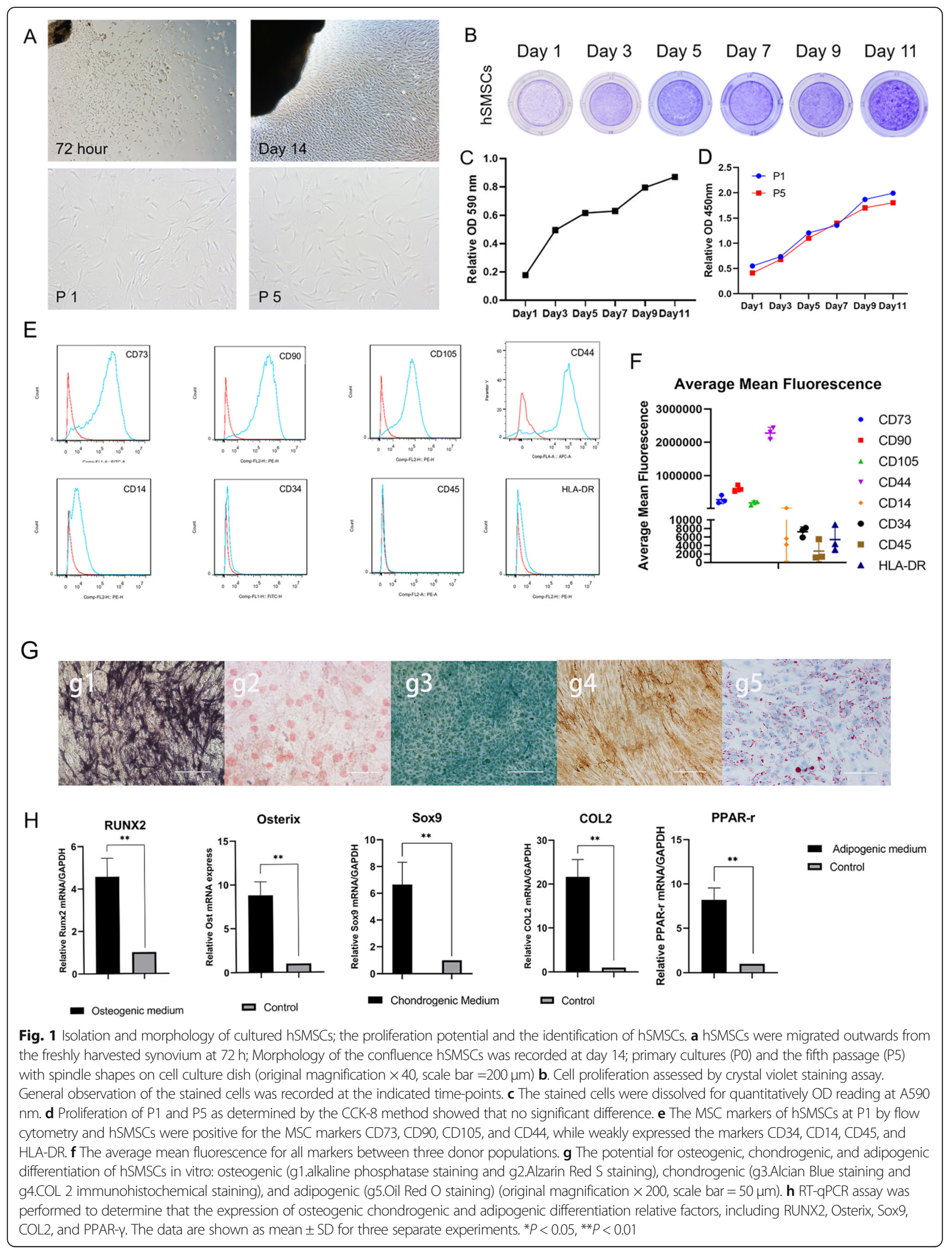




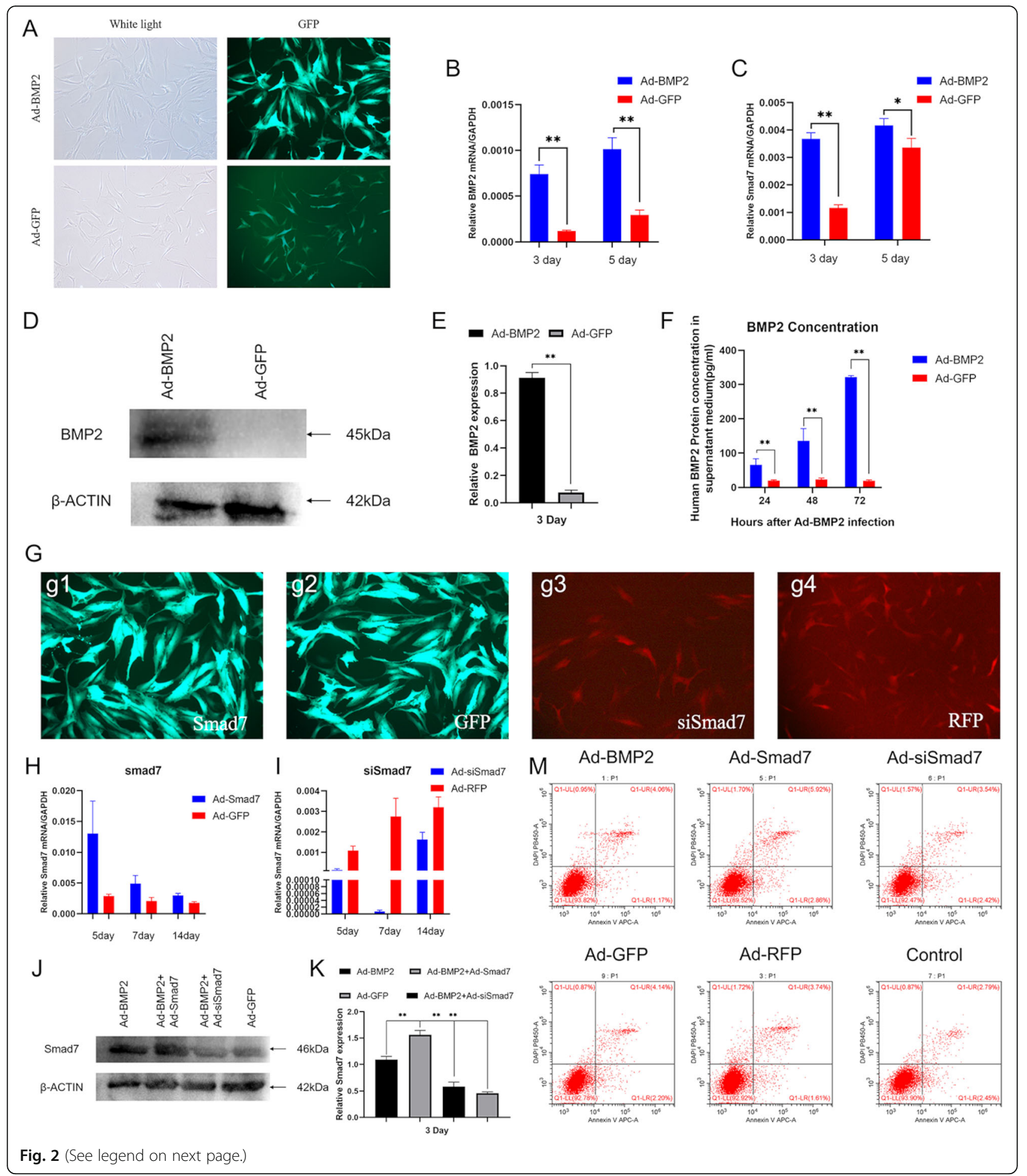




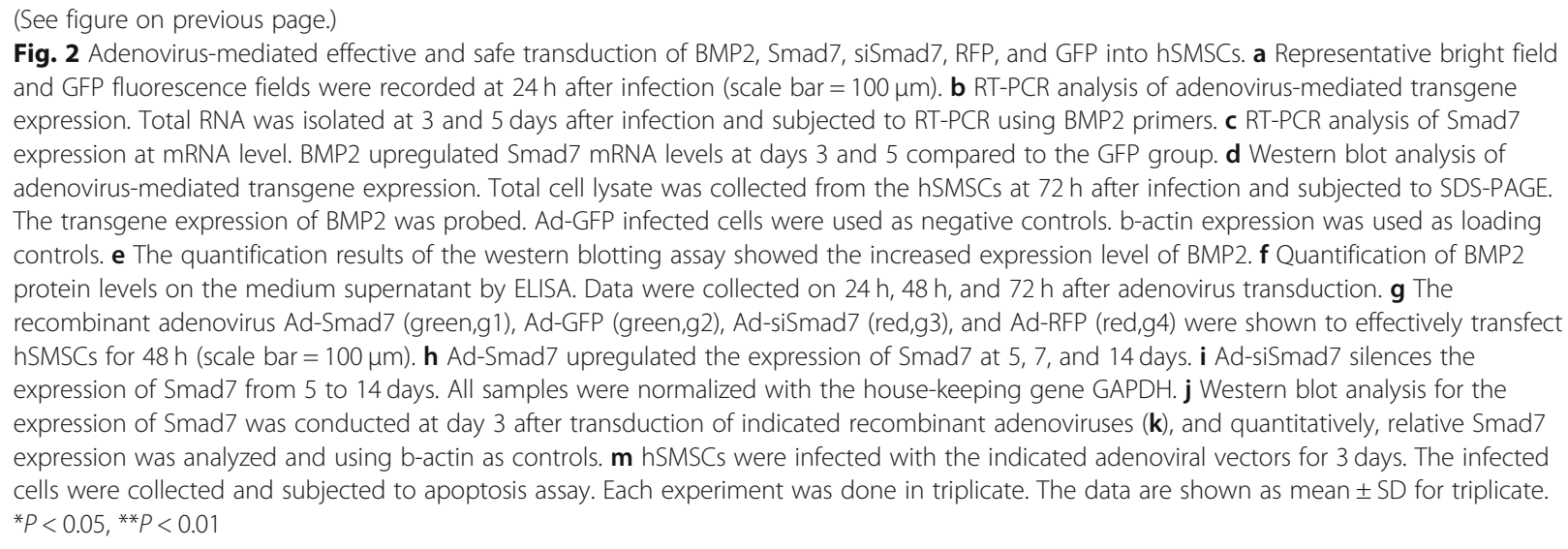

\section{hSMSCs have less osteogenic differentiation potential} in vitro

To assess the BMP2-induced osteogenic differentiation potential of hSMSCs compared with other MSCs, we used C3H10T1/2 cells and immortalized mouse adipose-derived MSCs (iMADs) (Fig. 3a). The cells were infected with the indicated adenovirus vectors. First, we examined the ALP activities by ALP staining at day 5, which indicated early osteogenic differentiation activity (Fig. 3b). Compared with that of hSMSCs, the ALP activity of C3H10T1/ 2 cells and iMads was considerably increased after BMP2 induction. Moreover, ALP quantitative analysis on day 5 showed that the ALP activity of the hSMSC group was dramatically lower than that of the other BMP2-treated groups (Fig. 3c). Second, Alizarin Red S staining was used to examine calcium deposition, which is one of the indicators of late osteogenic differentiation $[6,32]$. The results showed that hSMSCs also had less calcium deposition than C3H10T1/2 cells and iMads at 14 days (Fig. 3d,e). Quantitative data of osteogenic-related gene expression detected by RT-PCR showed that RUNX2, Osterix, and Osteocalcin were significantly increased 7 days after BMP2 treatment (Fig. 3f). hSMSCs showed less osteogenic gene expression than other groups. Taken together, hSMSCs exhibit lower BMP2-induced osteogenic differentiation potential.

\section{Smad7 reduces BMP2-induced chondrogenic differentiation and enhances hypertrophic differentiation} To confirm the function of Smad7 during BMP2induced chondrogenic differentiation and hypertrophic differentiation during hSMSC chondrogenesis, we harvested adenovirus-infected hSMSCs and seeded them as micromasses; these micromasses were cultured under chondrogenic conditions for 7 days and then cultured under hypertrophic medium conditions for 7 days $[10,44,45]$ (Fig. 4a). Alcian blue staining revealed that the level of sulfate glycosaminoglycans in the Smad7 silencing group was remarkably higher than that in the other groups and that in the Smad7 overexpression group was relatively lower than that in the BMP2 group on day 7 (Fig. 4b). Col2a1 is one of the most important molecular markers for chondrogenesis [2]. The immunohistochemical analysis results revealed that silencing $\mathrm{Smad} 7$ could significantly promote COL2 expression, while overexpressing Smad7 could inhibit BMP2-induced COL2 expression (Fig. $4 c, f)$. The RT-PCR data revealed that the successfully transfected BMP2 groups had remarkably higher expression of chondrogenic marker genes, including Sox9, COL2, and Aggrecan, than the control group, while the chondrogenic marker gene expression in the silenced Smad7 group was most significantly increased during the chondrogenic differentiation process. Conversely, the chondrogenic marker gene expression in the Smad7 overexpression group was decreased compared with that in the BMP2 group (Fig. 4g).

To confirm the role of Smad7 in hypertrophic differentiation, Col X and MMP13 hypertrophic markers were detected by immunohistochemical staining [46]. The protein expression of $\mathrm{Col} \mathrm{X}$ and MMP13 was clearly more upregulated in the BMP2 + Smad7 group at day 14 than in the other groups; the BMP2 + siSmad7 group showed lower protein expression levels than the BMP2 group and the BMP2 + Smad7 group but higher protein expression than the GFP group (Fig. 4d,e,f). Similarly, the RT-qPCR results revealed that the mRNA levels of RUNX2, Col X, and MMP13 in the BMP2 + siSmad7 group were significantly upregulated at day 14 compared to those in the BMP2 group (Fig. 4h).

In summary, these results indicate that $\operatorname{Smad} 7$ has a negative effect on chondrogenic differentiation-related factors and a positive effect on hypertrophic differentiation-related factors at the mRNA and protein expression levels. 







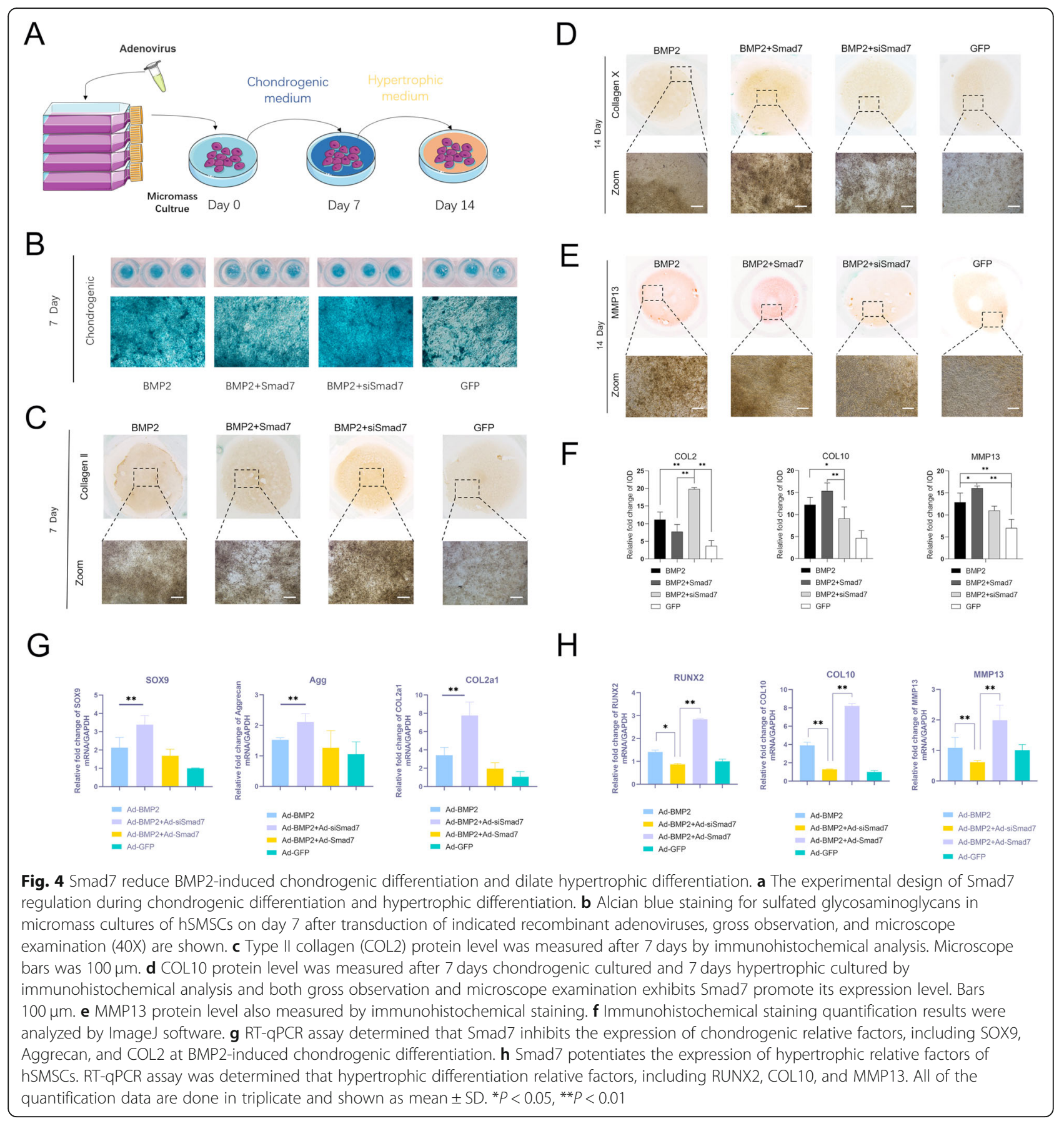

\section{Silencing Smad7 expression promotes chondrogenesis of the ectopic PPCNg-hSMSC composite and inhibits endochondral ossification in vivo}

We further examined the effect of combined PPCNg treatment on BMP2-induced chondrogenesis in vivo [9, 10, 13]. As a thermoresponsive macromolecule, PPCNg can provide a better in vivo microenvironment for hSMSCs [25]. The physical appearance of PPCNg remains liquid at $4{ }^{\circ} \mathrm{C}$ and quickly gels to form a solid scaffold at $37^{\circ} \mathrm{C}$ [44] (Fig. 5a). We mixed adenovirus- infected hSMSCs and PPCNg at $4{ }^{\circ} \mathrm{C}$ and implanted them subcutaneously into nude mice (Fig. 5b). We found that PPCNg alone and PPCNg with cells transduced with Ad-GFP, Ad-Smad7, or Ad-siSmad7 alone failed to form any detectable masses (data not shown). The general observation and micro-computed tomography (micro-CT) results showed that compared with that in the BMP2 group, the volume of ectopic mass in the BMP2 + siSmad7 group was increased (Fig. 5c,d). Quantitative analysis of bone histomorphology showed that compared 


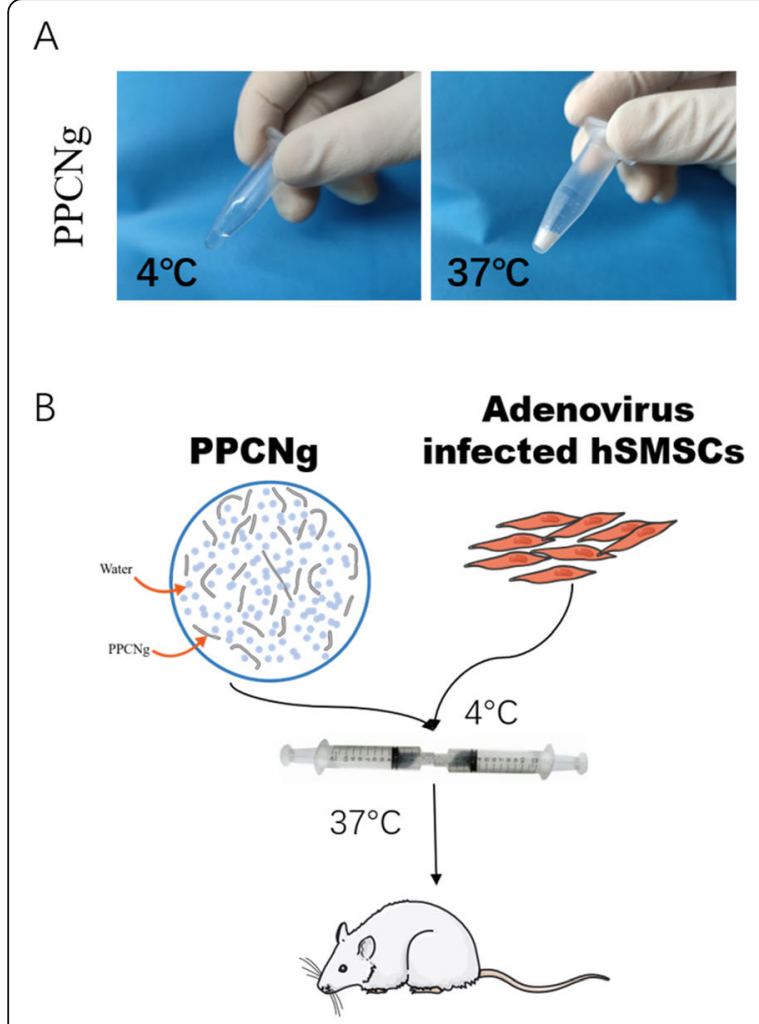

C
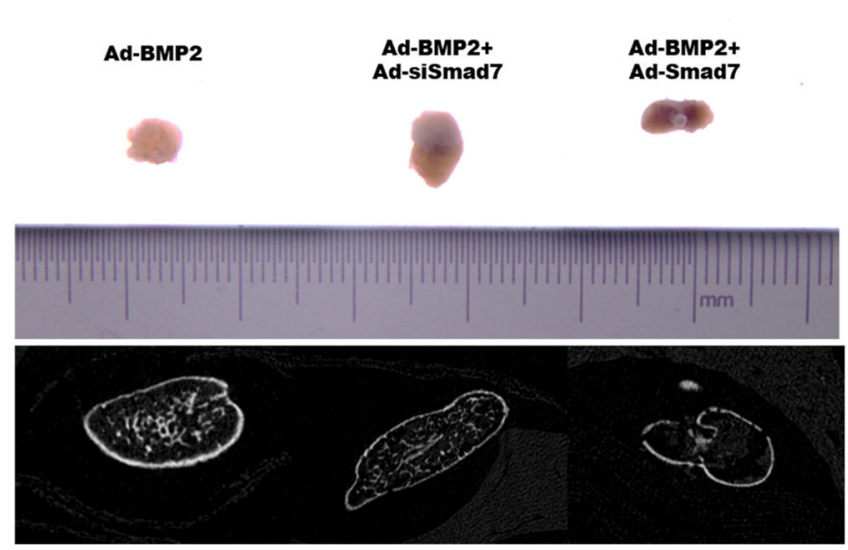

D

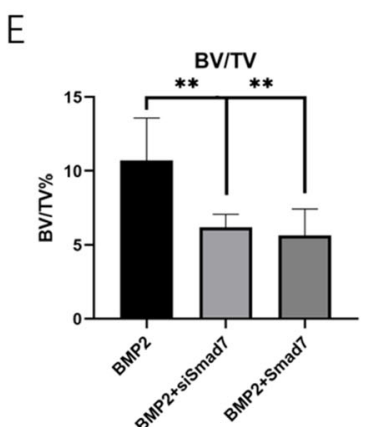

Fig. 5 Silencing Smad7 enhances the BMP2 induced subcutaneous ectopic cartilage formation (a). Before and after thermal-induced gelation of PPCNg alone, respectively. b Illustrative diagram showing that hSMSCs treated as experimental design seeded on the scaffolds was implanted into the subcutaneous space of the nude mice and harvested for analysis after 4 weeks. c Macrographic images of ectopic masses. The micro- $C T$ scanning to determine the surface and the volume of mass. $\mathbf{d}$ The retrieved masses were subjected to microCT analysis: Quantification results of the relative values of $\mathrm{BV} / \mathrm{TV}$ were analyzed. These data are shown as mean $\pm \mathrm{SD}$ repeat triplicate. ${ }^{*} P<0.05,{ }^{*} P<0.01$

with the BMP2 + Smad7 group and BMP2 + siSmad7 group, the bone volume/total volume (BV/TV) in the BMP2 group was significantly increased (Fig. 5e).

Histological examination revealed that the BMP2 + siSmad7 group had more mature chondrocytes and a few hypertrophic chondrocytes (Fig. 6a). In the BMP2 group, trabecular bone and vessel invasion were detected, suggesting that endochondral ossification had already started. The Smad7 group showed a large amount of undifferentiated hSMSCs. In immunohistochemical staining, the BMP2 group stained positive for COL2 and the fibroblast marker COL1. The BMP2 + siSmad7 group also stained positive for COL2, and negative for COL1, suggesting that stable mature cartilage was formed (Fig. 6b, d, e).

Based on immunofluorescence staining, we found that MMP13 and COL10 were also decreased in the BMP2 + siSmad7 group (Fig. 6c,f,g). These in vivo findings further confirm the chondrogenesispromoting effect of combined $\mathrm{PPCNg}$ and silenced Smad7 on BMP2-induced differentiation of hSMSCs.

\section{Discussion}

BMP2 induces not only cartilage formation but also endochondral ossification, although the precise mechanisms remain to be fully understood [9, 10, 13]. Endochondral ossification is important for the development, growth, and repair of the long bones [2]. However, endochondral ossification is the main obstacle of cartilage tissue engineering because it damages the extracellular matrix of cartilage $[11,12]$. BMP2-induced cartilage formation has been reported to be a possible strategy for cartilage repair. However, endochondral ossification during BMP2-induced differentiation still cannot be ignored and must be inhibited for further application of BMP2mediated cartilage repair $[9,10,13]$. In this study, we aimed to achieve ideal chondrogenesis by improving three elements: the use of human synovial-derived MSCs, silencing Smad7 expression, and implanting injectable thermosensitive cell scaffolds.

Mesenchymal stromal cells can easily be isolated from different tissues, and in vitro stimulation by cytokines commonly leads to chondrogenic differentiation [18]. Compared with bone marrow-derived MSCs (BMSCs) 


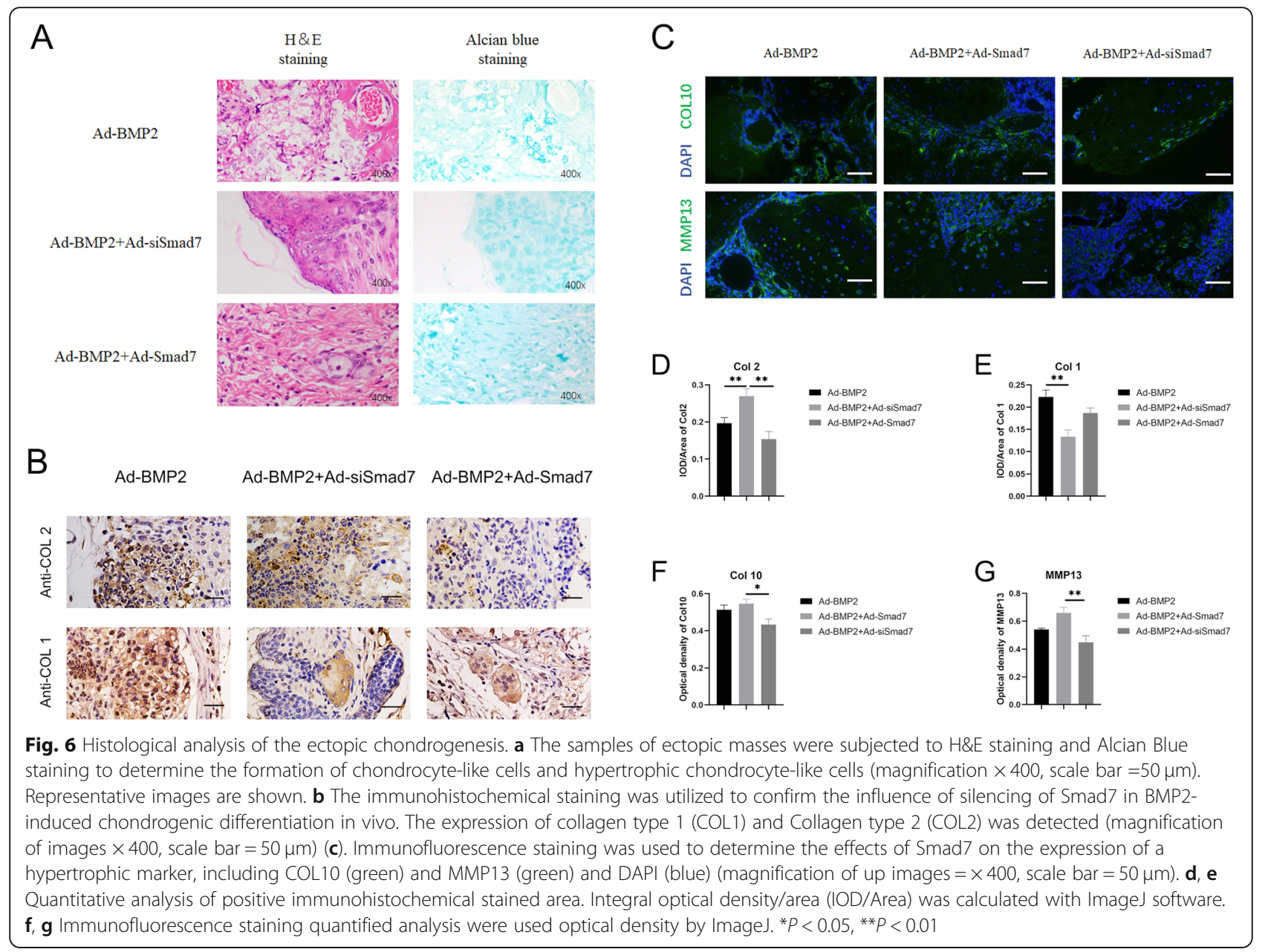

and synovial-derived stromal cells exhibit relatively poor potential for osteogenic differentiation [19]. In recent years, De Bari et al. found that adult synovium-derived Gdf5-positive cells failed to mineralize in vitro but still had the potential for chondrogenic differentiation [47]. This result indicates that endochondral ossification is difficult in hSMSCs. In addition, hSMSCs possess certain advantages, including low immunogenicity in vivo and powerful regenerative capabilities in vitro $[7,20,26,27]$. In the present study, primary human synovial mesenchymal stromal cells from the knee joint synovial membrane exhibited high proliferative activity and multidirectional differentiation potential. Moreover, these primary cells were identified by surface markers. However, we found that during BMP2-induced hSMSC differentiation in vitro, ALP activity was significantly increased. It also mineralized when cultured in osteogenic differentiation medium at day 14. The results suggest that BMP2induced osteogenesis ability is still powerful in hSMSCs. In vivo, hSMSCs were also capable of endochondral ossification differentiation induced by BMP2. Even so, compared with adipose tissue-derived stem cells (iMads) and
C3H10T1/2 mesenchymal stem cells of embryonic origin, hSMSCs have reduced osteogenic differentiation capacity. Our results indicate that among the most commonly studied MSCs, synovial stromal cells are the most specific for chondrogenic differentiation.

Smad7 may be the key to inhibiting endochondral ossification and affecting chondrogenesis [22, 23]. Our results revealed that Smad7 is upregulated in BMP2 overexpressing hSMSCs. As one of the two inhibitory Smad proteins, Smad7 is well known to inhibit TGF- $\beta$ pathways in a variety of mechanisms [21, 48]. Previous studies showed that overexpressing Smand7 disturbed mesenchymal condensation decreased chondrocyte proliferation and inhibited chondrocyte maturation [22]. In the endochondral ossification process of embryonic chondrogenesis, Smad7 knockout mice showed both skeletal defects and shortened hypertrophic zones in growth plates [24]. On the other hand, the exogenous expression of BMP2 and Smad7 by adenovirus transfection decreased the length of the hypertrophic zone of the growth plate in fetal mouse tibia compared with transfected BMP2 only [9]. The effect of silencing Smad7 
in BMP2-induced hSMSCs is still not reported. In this study, we used adenovirus transfection to significantly decrease Smad7 expression in hSMSCs to obtain a stable chondrocyte phenotype. In the chondrogenic differentiation experiment, silencing Smad7 expression significantly increased chondrogenic marker gene expression such as COL2a1 and SOX9, in hSMSCs. Furthermore, in the hypertrophic differentiation experiment, the Smad7silenced group had lower expression of hypertrophic chondrocyte markers, such as $\mathrm{Col} \mathrm{X}$ and MMP13, than the BMP2-induced only group. This result indicates that silencing Smad7 is a feasible strategy to increase chondrogenesis and matrix accumulation while inhibiting chondrocyte hypertrophic factor expression and endochondral ossification.

The cell attachment point and growth space are also indispensable for cartilage engineering [2, 3]. The subcutaneous transplantation of hSMSCs is limited by insufficient nutrient supply and irregular cell growth space, so we used PPCNg to improve this situation. This scaffold was mixed with a biodegradable citrate-based thermosensitive macromolecule, poly(polyethyleneglycol citrate-co- $N$-isopropylacrylamide) (PPCN), with gelatine (PPCN-g) [25]. The effectiveness of cell adhesion and the survival properties of MSCs have been proven by previous experiments in BMP9-induced osteogenic differentiation [40]. There are still no reports regarding the application of PPCN-g in MSC chondrogenic differentiation. We found that PPCN-g effectively promoted hSMSC chondrogenic differentiation in vivo. In ectopic hSMSC masses, the Smad7 silencing group had not only an increased number of COL2-positive cells but also a reduced number of hypertrophic chondrocytes and downregulated expression of the fibroblast marker COL1 and hypertrophic markers COL10 and MMP13. Compared with the BMP2 group, there was no obvious trabecular bone formation or new blood vessel invasion in the Smad7 silencing group. The standard endochondral ossification process is distinct from the in vitro experiment because of the lack of vascular invasion. Thus, the in vivo results have more practical value as a reference. The results strongly suggest that simultaneous application of hSMSCs, PPCN-g, and Smad7 knockdown can promote BMP2-induced chondrogenic differentiation and maintain the chondrocyte phenotype.

\section{Conclusion}

In summary, our findings suggested that simultaneous application of hSMSCs, PPCNg, and Smad7 knockdown can promote BMP2-induced chondrogenic differentiation and maintain stable chondrocyte phenotype. Thus, it is conceivable that this method may be exploited as a novel strategy to treat cartilage injuries.

\section{Supplementary Information}

The online version contains supplementary material available at https://doi. org/10.1186/s13287-021-02202-2.

Additional file 1: Supplementary Table 1. Primer sequence of the target genes. Supplementary. Figure 1. beta Galactosidase Staining of hSMSCs after adenovirus infection. Supplementary Figure 3.

Angiogenesis of ectopic masses.

\section{Abbreviations}

hSMSCs: Human synovial-derived mesenchymal stromal cells; BMP2: Bone morphogenetic protein 2; Smad7: SMAD Family Member 7; ITS: Insulintransferrin-sodium; FBS: Fetal bovine serum; DMEM: Dulbecco's modified Eagle's medium; iMads: Immortalized mouse adipose-derived mesenchymal cells; PPCNg: Polyethyleneglycol citrate-co-N-isopropylacrylamide with gelatin; GFP: Green fluorescent protein; RFP: Red Fluorescent protein; CCK8: Cell Counting Kit-8; SOX9: SRY-Box Transcription Factor 9; COL1: Collagen type I; COL2: Collagen type II; COL2a1: Collagen type II alpha 1 chain;

COL10: Collagen type X; MMP13: Matrix metallopeptidase 13; Agg: Aggrecan; ALP: Alkaline phosphatase; PBS: Phosphate-buffered saline; RUNX2: Runtrelated transcription factor 2

\section{Acknowledgements}

We are grateful for the Biomedical Materials Science, School of Biomedical Engineering, Third Military Medical University, Chongqing, and all persons in this department. We greatly thank Pro. Tong-Chuan He (Molecular Oncology Laboratory, Department of Surgery, University of Chicago Medical Center) and Pro. Shiwu Dong (Department of Biomedical Materials Science, School of Biomedical Engineering, Third Military Medical).

\section{Authors' contributions}

$\mathrm{WH}$ and $\mathrm{CZ}$ conceived and designed the experiments. PX, ZZ, DC, and $\mathrm{YZ}$ performed the experiments and collected the data. JL, QC, and HC analyzed the data. $\mathrm{CZ}$ and $\mathrm{WH}$ contributed to the reagents, materials, and analysis tools. PX, CZ, and WH wrote the manuscript. The authors read and approved the manuscript.

\section{Funding}

The reported work was supported by the National Natural Science Foundation of China (NSFC) (nos. 81371972, 81572142, and 81972069). Funding sources were not involved in the study design, in the collection, analysis, and interpretation of data; in writing of the report; and in the decision to submit the paper for publication.

\section{Availability of data and materials}

The datasets used and/or analyzed during the current study are available from the corresponding author on reasonable request.

\section{Ethics approval and consent to participate}

The animal study was reviewed and approved by the Ethical Committee of The First Affiliated Hospital of Chongqing Medical University. The human study was approved by the Research Ethics Committee of the First Affiliated Hospital of Chongqing Medical University. All participants signed informed consent on a voluntary basis.

\section{Consent for publication}

Not applicable

Competing interests

The authors declare that they have no competing interests.

Received: 19 October 2020 Accepted: 31 January 2021

Published online: 15 February 2021

\section{References}

1. Sophia Fox Alice J, Asheesh B, Rodeo Scott A. The basic science of articular cartilage: structure, composition, and function. Sports Health. 2009;1:461-8.

2. Makris Eleftherios A, Gomoll Andreas H, Malizos Konstantinos N, et al. Repair and tissue engineering techniques for articular cartilage. Nat Rev Rheumatol. 2015;11:21-34. 
3. Azizeh RDB, Soraya B, Hamid TN, et al. An overview of various treatment strategies, especially tissue engineering for damaged articular cartilage. Artif Cells Nanomed Biotechnol. 2020;48:1089-104.

4. Armiento Angela R, Mauro A, Stoddart Martin J. Articular fibrocartilage - why does hyaline cartilage fail to repair? Adv Drug Deliv Rev. 2019;146:289-305.

5. Danisovic L, Varga I, Polak S. Growth factors and chondrogenic differentiation of mesenchymal stem cells. Tissue Cell. 2012;44:69-73.

6. Geiger M, Li RH, Friess W. Collagen sponges for bone regeneration with rhBMP-2. Adv Drug Deliv Rev. 2003;55:1613-29.

7. Kovermann Nikolas J, Valentina B, Elena DB, et al. BMP2 and TGF- $\beta$ cooperate differently during synovial-derived stem-cell chondrogenesis in a dexamethasone-dependent manner. Cells. 2019;8 undefined.

8. Addisu M, Buchowski Jacob M, Zebala Lukas P, et al. High-dose rhBMP-2 for adults: major and minor complications: a study of 502 spine cases. J Bone Joint Surg Am. 2013;95:1546-53.

9. Chen Z, Wei J, Nian Z, et al. Sox9 augments BMP2-induced chondrogenic differentiation by downregulating Smad7 in mesenchymal stem cells (MSCs). Genes Dis. 2017:4:229-39.

10. Liao J, Hu N, Nian Z, et al. Sox9 potentiates BMP2-induced chondrogenic differentiation and inhibits BMP2-induced osteogenic differentiation. PLoS One. 2014;9:e89025.

11. Nian Z, Qi L, Lin X, et al. BMP2 induces chondrogenic differentiation, osteogenic differentiation and endochondral ossification in stem cells. Cell Tissue Res. 2016;366:101-11.

12. Murphy MP, Koepke LS, Lopez MT, Tong X, Ambrosi TH, Gulati GS, Marecic O, Wang Y, Ransom RC, Hoover MY, Steininger H, Zhao L, Walkiewicz MP, Quarto N, Levi B, Wan DC, Weissman IL, Goodman SB, Yang F, Longaker MT, Chan CKF. Articular cartilage regeneration by activated skeletal stem cells. Nat Med. 2020;26(10):1583-92. https://doi.org/10.1038/s41591-020-1013-2 Epub 2020 Aug 17. PMID: 32807933; PMCID: PMC7704061.

13. Dai G, Xiao H, Zhao C, Chen H, Liao J, Huang W. LncRNA H19 regulates BMP2-induced hypertrophic differentiation of mesenchymal stem cells by promoting Runx2 phosphorylation. Front Cell Dev Biol. 2020;8:580. Published 2020 Jul 29. https://doi.org/10.3389/fcell.2020.00580.

14. Lu Y, Zhou L, Wang L, He S, Ren H, Zhou N, Hu Z. The role of SIRT1 in BMP2-induced chondrogenic differentiation and cartilage maintenance under oxidative stress. Aging (Albany NY). 2020;12(10):9000-13. https://doi org/10.18632/aging.103161 Epub 2020 May 22. PMID: 32445555; PMCID: PMC7288925.

15. Sophie R, Emmanuel B, Marie M. Non-viral gene activated matrices for mesenchymal stem cells based tissue engineering of bone and cartilage. Biomaterials. 2016;104:223-37.

16. Urszula K, Agnieszka K, Katarzyna F, et al. Similarities and differences between mesenchymal stem/progenitor cells derived from various human tissues. World J Stem Cells. 2019;11:347-74.

17. Kendrick T, Bridget $Z$, Karl R, et al. In vivo synovium-derived mesenchymal stem cell transplantation in cartilage regeneration: a PRISMA review of studies. Front Bioeng Biotechnol. 2019;7:314

18. Dennis MG, Baboolal Thomas G, Elena J. Native joint-resident mesenchymal stem cells for cartilage repair in osteoarthritis. Nat Rev Rheumatol. 2017;13: 719-30

19. Farida D, Claire B, Thomas $H$, et al. Transcriptional profiles discriminate bone marrow-derived and synovium-derived mesenchymal stem cells. Arthritis Res Ther. 2005;7:R1304-15.

20. Wataru A, Kutcher Josh J, Roman K, et al. Clonal analysis of synovial fluid stem cells to characterize and identify stable mesenchymal stromal cell/ mesenchymal progenitor cell phenotypes in a porcine model: a cell source with enhanced commitment to the chondrogenic lineage. Cytotherapy. 2014:16:776-88

21. Miyazawa K, Miyazono K. Regulation of TGF- $\beta$ family signaling by inhibitory Smads. Cold Spring Harb Perspect Biol. 2017;9(3):a022095. https://doi.org/1 0.1101/cshperspect.a022095 PMID: 27920040; PMCID: PMC5334261.

22. Takao I, Junko M, Hideki Y, et al. Smad7 inhibits chondrocyte differentiation at multiple steps during endochondral bone formation and down-regulates p38 MAPK pathways. J Biol Chem. 2008;283:27154-64.

23. Blaney Davidson Esmeralda N, Vitters Elly L, van den Berg Wim B, et al. TGF beta-induced cartilage repair is maintained but fibrosis is blocked in the presence of Smad7. Arthritis Res Ther. 2006;8:R65.

24. Estrada Kristine D, Weiguang W, Retting Kelsey N, et al. Smad7 regulates terminal maturation of chondrocytes in the growth plate. Dev Biol. 2013; 382:375-84
25. Ye J, Wang J, Zhu Y, et al. A thermoresponsive polydiolcitrate-gelatin scaffold and delivery system mediates effective bone formation from BMP9transduced mesenchymal stem cells. Biomed Mater. 2016;11(2):025021. Published 2016 Apr 21. https://doi.org/10.1088/1748-6041/11/2/025021.

26. Tao SC, Yuan T, Zhang YL, Yin WJ, Guo SC, Zhang CQ. Exosomes derived from miR-140-5p-overexpressing human synovial mesenchymal stem cells enhance cartilage tissue regeneration and prevent osteoarthritis of the knee in a rat model. Theranostics. 2017;7(1):180-95. Published 2017 Jan 1. https:// doi.org/10.7150/thno.17133.

27. Shintani N, Siebenrock KA, Hunziker EB. TGF-b1 enhances the BMP-2induced chondrogenesis of bovine synovial explants and arrests downstream differentiation at an early stage of hypertrophy. PLoS One. 2013:8:e53086.

28. Song D, Zhang F, Reid RR, et al. BMP9 induces osteogenesis and adipogenesis in the immortalized human cranial suture progenitors from the patent sutures of craniosynostosis patients. J Cell Mol Med. 2017;21(11): 2782-95. https://doi.org/10.1111/jcmm.13193.

29. Shu Y, Yang C, Ji X, et al. Reversibly immortalized human umbilical cordderived mesenchymal stem cells (UC-MSCS) are responsive to BMP9induced osteogenic and adipogenic differentiation. J Cell Biochem. 2018; 119(11):8872-86. https://doi.org/10.1002/jcb.27140.

30. Cao J, Chen $X$, Jiang L, et al. DJ-1 suppresses ferroptosis through preserving the activity of S-adenosyl homocysteine hydrolase. Nat Commun. 2020;11(1): 1251. Published 2020 Mar 6. https://doi.org/10.1038/s41467-020-15109-y.

31. Zhu X, Gong Y, Liu Y, et al. Ru@CeO2 yolk shell nanozymes: oxygen supply in situ enhanced dual chemotherapy combined with photothermal therapy for orthotopic/subcutaneous colorectal cancer, 2020 Feb 28. Biomaterials. 2020;242:119923. https://doi.org/10.1016/j.biomaterials.2020.119923.

32. Li Y, Liu Z, Tang Y, et al. Schnurri-3 regulates BMP9-induced osteogenic differentiation and angiogenesis of human amniotic mesenchymal stem cells through Runx2 and VEGF. Cell Death Dis. 2020;11(1):72. Published 2020 Jan 29. https://doi.org/10.1038/s41419-020-2279-5.

33. Tiziana S, Gianfranco P, Umberto G, cells C t w m s. An Update. Cell Transplant. 2016:25:829-48.

34. Zhao C, Wu N, Deng F, et al. Adenovirus-mediated gene transfer in mesenchymal stem cells can be significantly enhanced by the cationic polymer polybrene. PLoS One. 2014;9(3):e92908.

35. Deng Y, Zhang J, Wang Z, et al. Antibiotic monensin synergizes with EGFR inhibitors and oxaliplatin to suppress the proliferation of human ovarian cancer cells. Sci Rep. 2015:5:17523.

36. Fan J, Wei Q, Liao J, et al. Noncanonical Wnt signaling plays an important role in modulating canonical Wnt-regulated stemness, proliferation and terminal differentiation of hepatic progenitors. Oncotarget. 2017;8(16): $27105 \mathrm{e} 27119$

37. Zhang F, Li Y, Zhang H, et al. Anthelmintic mebendazole enhances cisplatin's effect on suppressing cell proliferation and promotes differentiation of head and neck squamous cell carcinoma (HNSCC). Oncotarget. 2017;8(8):12968e12982.

38. Sun $M$, Hu L, Wang S, Huang T, Zhang M, Yang M, Zhen W, Yang D, Lu W, Guan M, Peng S. Circulating microRNA-19b identified from osteoporotic vertebral compression fracture patients increases bone formation. J Bone Miner Res. 2020;35(2):306-16

39. Hou Y, Cao X, Hu X, Li X, Shi X, Wang H, Peng C, Li J, Li J, Li Q, Wu C. Xiao XCMHX008, a PPARY partial agonist, enhances insulin sensitivity with minor influences on bone loss. Genes Dis. 2018:5(3):290-9.

40. Zhao C, Zeng Z, Qazvini NT, et al. Thermoresponsive citrate-based graphene oxide scaffold enhances bone regeneration from BMP9-stimulated adiposederived mesenchymal stem cells. ACS Biomater Sci Eng. 2018;4(8):2943-55. https://doi.org/10.1021/acsbiomaterials.8b00179.

41. Qiu J, Hirschi KK. Endothelial cell development and its application to regenerative medicine. Circ Res. 2019;125:489-501.

42. Park MH, et al. Differential expression patterns of Runx2 isoforms in cranial suture morphogenesis. J Bone Miner Res. 2001;16:885-92.

43. Alessio N, Stellavato A, Squillaro T, Del Gaudio S, Di Bernardo G, Peluso G De Rosa M, Schiraldi C, Galderisi U. Hybrid complexes of high and low molecular weight hyaluronan delay in vitro replicative senescence of mesenchymal stromal cells: a pilot study for future therapeutic application. Aging (Albany NY). 2018;10(7):1575-85. https://doi.org/10.18632/aging.1014 93 PMID: 30001217; PMCID: PMC6075440.

44. Zhao C, Miao Y, Cao Z, et al. MicroRNA-29b regulates hypertrophy of murine mesenchymal stem cells induced toward chondrogenesis 
[published online ahead of print, 2019 Jan 16]. J Cell Biochem. 2019. https:// doi.org/10.1002/jcb.28161.

45. Yang B, Guo H, Zhang Y, Dong S, Ying D. The microRNA expression profiles of mouse mesenchymal stem cell during chondrogenic differentiation. BMB Rep. 2011;44(1):28-33. https://doi.org/10.5483/BMBRep.2011.44.1.28.

46. Mueller MB, Tuan RS. Functional characterization of hypertrophy in chondrogenesis of human mesenchymal stem cells. Arthritis Rheum. 2008; 58(5):1377-88. https://doi.org/10.1002/art.23370.

47. Roelofs AJ, Zupan J, Riemen AHK, et al. Joint morphogenetic cells in the adult mammalian synovium. Nat Commun. 2017;8:15040. Published 2017 May 16. https://doi.org/10.1038/ncomms15040.

48. Yan X, Liu Z, Chen Y. Regulation of TGF-beta signaling by Smad7. Acta Biochim Biophys Sin Shanghai. 2009;41(4):263-72. https://doi.org/10.1093/a bbs/gmp018.

\section{Publisher's Note}

Springer Nature remains neutral with regard to jurisdictional claims in published maps and institutional affiliations.

Ready to submit your research? Choose BMC and benefit from:

- fast, convenient online submission

- thorough peer review by experienced researchers in your field

- rapid publication on acceptance

- support for research data, including large and complex data types

- gold Open Access which fosters wider collaboration and increased citations

- maximum visibility for your research: over $100 \mathrm{M}$ website views per year

At $\mathrm{BMC}$, research is always in progress.

Learn more biomedcentral.com/submissions 